\title{
Genomic sequencing in acutely ill infants: what will it take to demonstrate clinical value?
}

\author{
Scott D. Grosse, $\mathrm{PhD}^{1}$ and Lauge Farnaes, $\mathrm{MD}, \mathrm{PhD}^{2,3}$ \\ Genetics in Medicine (2019) 21:269-271; https://doi.org/10.1038/s41436-018-0124-3
}

Proponents of rapid genomic sequencing in infants in neonatal or pediatric intensive care units (NICU/PICUs), which includes both rapid genome sequencing (rGS) and rapid exome sequencing ( $\mathrm{rES}$ ), argue that it can deliver timely diagnostic results and enable life-changing clinical interventions. Moreover, it has been suggested that this application could serve as a "breakthrough" to the widespread clinical use of next generation sequencing (NGS) tests. ${ }^{1}$ On the other hand, payers might resist agreeing to pay for NGS in acutely ill infants for fear of establishing a precedent for reimbursement for NGS in much larger patient populations.

In this issue, a group of international experts has prepared a white paper that acknowledges the "great promise" of genome-wide sequencing (GWS) for acutely ill infants but emphasizes gaps in evidence. ${ }^{2}$ Friedman and colleagues lay out principles for establishing the clinical value of such testing. We agree that it is essential that a robust evidentiary base of benefits be developed to allow health-care providers and payers to make informed decisions on the implementation of NGS technologies in that patient population.

Friedman and colleagues propose that the first step in demonstrating clinical value of NGS in acutely ill children is the demonstration of analytic validity. Analytic validity is an important laboratory metric, and it is a crucial requirement for population screening to minimize false-positive and falsenegative results. However, for ill patients, the important criteria are whether testing identifies pathogenic variants that explain disease phenotypes and whether timely return of results affects patient management and outcomes. Given the complexity of assessing analytic validity for thousands of gene variants, we suggest that the key to broad clinical implementation of NGS in this patient population is demonstrated evidence of clinical utility.

Friedman et al. suggest that mortality is the most compelling summary measure of the value of NGS compared with standard of care in this population, but we are skeptical for two reasons. First, as the authors cogently explain, diagnosis of an untreatable condition based on NGS results may lead to cessation of painful and ultimately futile procedures. ${ }^{2}$ For those infants, early genomic diagnoses may lead to earlier death while simultaneously reducing suffering for the child and psychosocial harm to the parents through earlier transition to palliative care. Infants who have treatable conditions can benefit from initiation of appropriate treatment in terms of quality of life, but the extent to which treatment affects survival is not necessarily known. We propose reductions in total number of hospital days as a summary measure of nonfatal outcomes associated with timely genomic diagnoses in acutely ill infants.

Friedman and colleagues conclude, "Comparing diagnostic GWS to the current standard of care per case of serious genetic disease diagnosed can provide a practical means of assessing clinical value" and propose, "for a comparative study to provide the robust evidence that is needed, both costs and benefits will have to be collected in a rigorous prospective manner, and the results will need to be analyzed comprehensively." ${ }^{2}$ We concur. Indeed, several cohort studies and randomized trials have either been published or are underway that are tracking health outcomes and costs associated with genomic diagnoses in acutely ill children. In particular, evidence shows that short-term cost savings from a shortening of the diagnostic process and from reduced inpatient costs for those infants who experience substantial health improvements can more than pay for the cost of sequencing and clinical interpretation. We need to draw lessons from that emerging body of clinical research and discuss how such analyses can be replicated and extended to address long-term costs and health outcomes from both the health-care and societal perspectives. We propose that to address the societal implications of this technology, researchers assess spillover costs and benefits to patients and families. ${ }^{3}$ In addition, families may value a genetic diagnosis for a sick child even if the diagnosis is not of immediate clinical benefit. The "personal utility" of genomic diagnoses can reflect prognostic

\footnotetext{
${ }^{1}$ National Center on Birth Defects and Developmental Disabilities, Centers for Disease Control and Prevention, Atlanta, Georgia, USA; ${ }^{2}$ Rady Children's Institute for Genomic Medicine, San Diego, California, USA; ${ }^{3}$ Department of Pediatrics, University of California at San Diego, San Diego, California, USA. Correspondence: Scott D. Grosse (sgrosse@cdc. gov) 
value, the hope of a cure in the future, and increased knowledge of genetic risk for other family members, among other considerations. ${ }^{4,5}$

Stark and colleagues recently demonstrated improved care and net cost savings from singleton rES of critically ill infants and children cared for at the Royal Children's Hospital and Monash Children's Hospital in Melbourne, Australia. ${ }^{6}$ In this study 40 critically ill children aged 3 days to 4 years were enrolled with parental consent for rES based on clinical consensus of a possible genetic etiology. A genomic diagnosis was found at a median of 16 days for $52.5 \%$ of the 40 infants. A change in management occurred in $14(35 \%)$ of the patients sequenced, including 2 children who were redirected to palliative care. In 2 children, rES resulted in much more rapid diagnosis than was achieved through standard testing. Total cost of sequencing the cohort was AU $\$ 158,360$, and the total cost of all examinations was AU $\$ 281,143$. Estimated potential avoided cost of acute care based on reduced length of stay for 2 children who achieved earlier diagnosis with $\mathrm{rES}$ was AU\$543,178. One child with a riboflavin transporter defect was estimated to have had 115 PICU days with mechanical ventilation avoided with rES. Relative to the estimated costs associated with traditional diagnostic testing, net health-care savings were estimated at AU\$262,035.

Similarly, a study at the Rady Children's Institute of Genomic Medicine in San Diego, California retrospectively assessed 42 critically ill children less than 1 year old who received rGS. ${ }^{7}$ Of 42 patients sequenced, 18 received a diagnosis, of which 13 received significant changes in management as a result. In 7 (17\%) very ill infants, rGS was performed with interpretation in 2 days; the remaining 35 (83\%) families received rGS with interpretation in 5 days. Total cost for sequencing the 42 infants and, in 39 cases other family members, was $\$ 674,645$. The relatively high cost compared with the Melbourne study reflected the increased speed to interpretation, the use of rGS rather than rES, and the frequent performance of trios (child and two parents). If singleton (proband-only) sequencing had been conducted, the cost would have been about one-half as much. Cost savings were assessed in six patients for whom physicians agreed that medical changes were attributable to the rapid genomic diagnosis. The estimated total avoidable cost was $\$ 803,199$, with an aggregate reduction of 124 hospital days. A conservative estimate of net cost savings after subtracting the cost of rGS was $\$ 128,554$. That included savings of $\$ 327,506$ for a patient with Coffin-Siris syndrome associated with a $A R I D 1 B$ variant who was moved to comfort care when the diagnosis was made, thereby avoiding a planned 6-week stay in the NICU for antibiotic treatment. ${ }^{7}$ If the diagnosis and transition to palliative care had occurred substantially sooner, short-term cost savings could have been as much as an additional $\$ 1.2$ million. ${ }^{8}$ A child with Ohtara syndrome associated with a KCNQ2 variant (https:// www.radyfoundation.org/about-us/patient-stories/sebastiana) was diagnosed in 4 days, compared with 42 days for a child previously diagnosed using genome sequencing, avoiding 38 hospital days and $\$ 181,141$ in additional cost. ${ }^{7}$

A randomized trial of rGS would provide more definitive evidence of benefit, but it may not be feasible to enroll sufficient numbers of evidence for a trial to be adequately powered to test the hypothesis of clinical benefit. A trial in a NICU population is being conducted at Boston Children's Hospital as part of the BabySeq project under the Newborn Sequencing In Genomic medicine and public HealTh (NSIGHT) consortium funded by the National Human Genome Research Institute; ${ }^{9}$ no results have been released (http://www.genomes2people.org/babyseqproject/). The window to conduct trials in a NICU population may be closing as observational evidence of diagnostic benefit accumulates. A small trial of rGS in acutely ill infants intended to assess the impact on age at diagnosis was stopped early because of a high crossover rate in the control arm, the majority of whom received genomic sequencing, resulting from a loss of equipoise. ${ }^{10}$ Even though rGS is not yet standard of care, it may become increasingly difficult to enroll families in a trial in which some acutely ill infants are sequenced while others with similar symptoms are denied the same opportunity.

Real-world (observational) data is therefore likely to remain the primary source of information on the benefits of NGS in acutely ill infants. While both the Melbourne and San Diego studies showed net reductions in the short-term cost of acute hospital care, a complete analysis of economic benefits associated with a timely genomic diagnosis would require estimates of long-term health outcomes and healthcare costs. Further, spillover effects on parental psychological stress and the burden of informal care and lost work time are important for economic analyses from the societal perspective. ${ }^{3}$ The cost of supportive care for a child with a permanent neurological condition can comprise a large fraction of total economic cost. Initiatives to develop and link real-world data on cohorts of infants who undergo rapid NGS in NICU/PICU settings in multiple centers and their families' experiences could generate the kinds of data needed for complete assessments of health and economic outcomes. Similar initiatives have been proposed in cancer genetics. ${ }^{11}$

\section{DISCLOSURE}

Scott Grosse received travel support from the Rady Children's Institute of Genomic Medicine. Lauge Farnaes is an employee of the Rady Children's Institute of Genomic Medicine.

\section{REFERENCES}

1. Kingsmore SF, Petrikin J, Willig LK, et al. Emergency medical genomes: a breakthrough application of precision medicine. Genome Med. 2015:7:82.

2. Friedman JM, Bombard Y, Cornel MC, et al. Genome-wide sequencing in acutely-ill infants: genomic medicine's critical application? Genet Med. 12 June 2018. https://doi.org/10.1038/s41436-018-0055-z

3. Tilford JM, Payakachat N. Progress in measuring family spillover effects for economic evaluations. Expert Rev Pharmacoecon Outcomes Res. 2015;15:195-198.

4. Bunnik EM, Janssens AC, Schermer MH. Personal utility in genomic testing: is there such a thing? J Med Ethics. 2015;41:322-326. 
5. Kohler JN, Turbitt E, Lewis KL, et al. Defining personal utility in genomics: a Delphi study. Clin Genet. 2017;92:290-297.

6. Stark Z, Lunke S, Brett GR, et al. Meeting the challenges of implementing rapid genomic testing in acute pediatric care. Genet Med. 15 May 2018. https://doi.org/10.1038/gim.2018.37

7. Farnaes L, Hildreth A, Sweeney NM, et al. Rapid whole-genome sequencing decreases infant morbidity and cost of hospitalization. NPJ Genom Med. 2018;3:10.

8. Sweeney NM, Nahas SA, Chowdhury S, et al. The case for early use of rapid whole genome sequencing in management of critically ill infants: late diagnosis of Coffin-Siris syndrome in an infant with left congenital diaphragmatic hernia, congenital heart disease and recurrent infections. Cold Spring Harb Mol Case Stud. 2018;4:a002469.

9. Berg JS, Agrawal PB, Bailey DB Jr., et al. Newborn sequencing in genomic medicine and public health. Pediatrics. 2017;139:e20162252.

10. Petrikin JE, Cakici JA, Clark MM, et al. The NSIGHT1-randomized controlled trial: rapid whole-genome sequencing for accelerated etiologic diagnosis in critically ill infants. NPJ Genom Med. 2018;3:6.

11. Agarwala $V$, Khozin $S$, Singal $G$, et al. Real-world evidence in support of precision medicine: clinico-genomic cancer data as a case study. Health Aff (Millwood). 2018;37:765-772. 